\title{
Populations of Extrasolar Giant Planets from Transit and Radial Velocity Surveys
}

\author{
Alexandre Santerne
}

\begin{abstract}
Transit and radial velocity surveys have deeply explored the population of extrasolar giant planets, with hundreds of objects detected to date. All these detections allow to understand their physical properties and to constrain their formation, migration, and evolution mechanism. In this chapter, the observed properties of these planets are presented along with the various populations identified in the data. The occurrence rates of giant exoplanets, as observed in different stellar environment by various surveys are also reviewed and compared. Finally, the presence and properties of the giant exoplanets are discussed in the regards of the properties of the host star. Over this chapter, the observational constraints are discussed in the context of the dominant planet formation, migration and evolution scenarios.
\end{abstract}

\section{Introduction}

Two decades of exploration of extrasolar giant planets (hereafter EGPs) with radial velocity and transit surveys, both from the ground and from space, have revolutionised our view on giant planets, in comparison with the solar system. At a time when Earth-sized exoplanets are discovered in the habitable zone of their star, many questions regarding the formation, migration, and evolution of EGPs are not yet fully understood. For instance, their dominant formation mechanisms is still debated: either by core-accretion (e.g. Mordasini et al. 2012) or disk instability (see Nayakshin 2017, for a review). The physical process causing the inflation of giant planets is also unclear (Baraffe et al.2014). Even the definition of what is an EGP, with respect to brown dwarfs is actively discussed (Schneider et al. 2011, Chabrier et al. 2014; Hatzes and Rauer 2015).

Alexandre Santerne

Aix Marseille Univ, CNRS, CNES, LAM, Marseille, France, e-mail: al lexandre.santerne@ lam.fr 
Nevertheless, hundreds of EGPs have been discovered and well characterised thanks to photometric surveys like Super-WASP (Pollacco et al.2006) and HATNet (Bakos et al. 2004) from the ground and CoRoT (Baglin et al. 2006) and Kepler (Borucki et al. 2009) from space, as well as spectroscopic surveys like with the CORALIE and HARPS (Mayor et al.2011), the SOPHIE (e.g. Hébrard et al.2016), and the Lick and Keck (e.g. Marcy et al.2005) instruments and observatories (for a more complete list of instruments and surveys, see the corresponding sections of this book). All these discoveries bring important insights into giant planet formation, migration and evolution. This chapter highlights the main interpretation of all the EGP discoveries, starting with a description of the different populations of EGP, then the occurrence rates of EGP as determined in different stellar environments, the relation between the presence and properties of EGPs with respect to their host star, and finally the conclusions.

\section{Different Populations of EGP}

To date, nearly 1000 EGPs have been detected and characterised by photometric and spectroscopic surveys. They are displayed in the Fig. 11 together with their distribution. From all these detections, there is a clear limit between giant planets and lower-mass, Neptune-like planets at about $0.1-0.2 \mathrm{M}_{4}$ (about $30-60 \mathrm{M}_{\oplus}$ ). In this regime, very few objects have been detected either by transit or RV. This cannot be explained by an observational bias. This lower-limit in mass for the giant planet is supported by the threshold at which the planetesimal starts the runaway accretion and also open a gap in the disk changing their migration from type I and type II (e.g. Crida and Bitsch 2017).

The upper-limit in mass, arbitrarily set at $\sim 30 \mathrm{M}_{4}$ in Fig. 1 corresponds to the one used by the NASA exoplanet archive (Akeson et al. 2013).

\section{Hot Jupiter}

Planets more massive than $0.1-0.2 \mathrm{M}_{4}$ are clearly distributed in two main clusters. The first cluster is very close to the star, with semi-major axis of less than $0.1 \mathrm{AU}$ (equivalent to about 10 days for sun-like stars). These are the so-called hot Jupiters as these planets are highly irradiated by their host star. The most typical member of this population is 51 Peg b (Mayor and Queloz 1995). This population of hot Jupiters has been deeply explored by ground-based photometric surveys with hundreds of detections. They are the easiest planets to detect, even within reach of amateur facilities (e.g. Santerne 2014). 


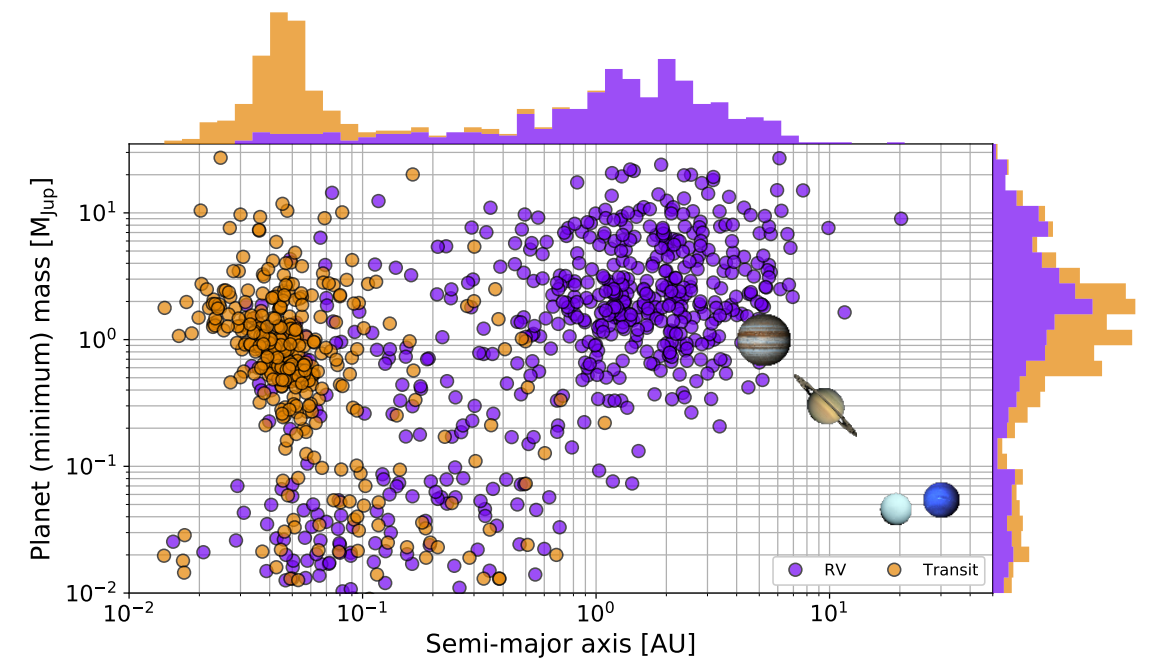

Fig. 1 Extrasolar planets discovered to date (source: NASA Exoplanet Archive) by the transit (orange marks) and radial velocity (violet marks) surveys. The planetary mass (sky-projected minimum mass in case of RV planets) is shown as function of the semi-major axis of the orbit. The top and right histograms represent the raw distribution of extrasolar planets in their semi-major axis and (minimum) mass, respectively. This reveals the two main populations of EGP and a transitional population where few objects have been found. Solar system planets are also present for comparison.

\section{Temperate/Cold Giants}

The second cluster is located at much larger separation, starting at about $0.4 \mathrm{AU}$ (about 100 days for sun-like stars) up to several AU. This population is composed by temperate to cold giants and includes planets like Jupiter. Transit surveys from the ground are not very sensitive to planets with periods greater than about 10 days and are not able to probe this population. Only space-based photometry lasting several years like Kepler was able to detect giant planets at a few hundreds days, but given their low transit probability (less than $0.5 \%$ at $1 \mathrm{AU}$ for a sun-like star), the number of temperate/cold giants detected in transit is small (Santerne et al.2016).

Note that the lack of planets detected beyond the orbit of Saturn is only due to observational bias. Two decades of spectroscopic observations allows for the detection of planets with periods up to typically 20 years. As a consequence, planets with orbital period longer than Saturn $(\sim 29$ years) present only partial orbits in the data which is therefore poorly determined for now. Radial velocity observations in the next decades will likely extend this population of planets towards larger separation, if these planets are common. Planets less massive than Saturn at a few AU are also not yet detected because of instrumental limitations. 


\section{Period-valley giants}

Between these two clusters resides a transition where relatively few EGP have been found. This transition is known as the period valley, first identified by Udry et al. (2003) based on radial velocity detections. If this transition population is clear in spectroscopic surveys (see also Udry and Santos 2007), it was first unconfirmed by the transit detections of the Kepler mission (Howard et al. 2012, Fressin et al. 2013), indicating only one, continuous population of EGPs. This period-valley was however confirmed in the Kepler detections by Santerne et al. (2016) after a systematic removal of false-positive contaminations in the sample using the SOPHIE spectrograph. This valley in the period distribution of EGPs is a strong indication that temperate/cold giants and hot Jupiters have different formation and/or migration mechanisms.

Nevertheless, this period-valley population appears narrower in the Kepler data (only restricted to within 10 - 20 days Santerne et al. 2016) compared to spectroscopic data (extended between 10 and about 100 days). One reason for this behaviour is that Kepler detected several EGPs with orbital periods between 20 and 100 days that belong to multiple planetary systems, like Kepler-9 (Holman et al. 2010), Kepler-51 (Masuda 2014), Kepler-89 (Weiss et al. 2013), and Kepler-117 (Bruno et al. 2015). These EGPs might have stopped their migration in the period valley and did not end as hot Jupiters because of their companion, as it is proposed for the pair Jupiter - Saturn in the solar system (Morbidelli and Crida 2007).

Note that the period valley is poorly explored by ground-based transit surveys as their sensitivity drops drastically above 10 days, i.e. at the upper limit of the hot jupiter population. Therefore, the relative weight between the hot-jupiter, the temperate/cold giants and the period-valley populations seen in Fig. 1 is strongly dominated by observing bias.

\section{Lack of Very-Short Period EGP}

Another clear behaviour observed with all the EGP detections is the lack of lowmass, hot, giant planets (less massive than Jupiter, down to super-Earth) orbiting very close to their star (up to 0.04AU, see Fig. 1). This desert is fully described in Mazeh et al. (2016). Two main reasons have been proposed to explain this desert. The first reasons is that low-mass EGPs in the desert are too much irradiated by their host star and consequently, they loose their atmosphere (as it is observed for some hot Jupiters, e.g. Vidal-Madjar et al. 2003). Thus, they migrate down in the mass - period diagramme. The remnants of photo-evaporated hot Jupiters could be short-period super-Earths (Valsecchi et al. 2014). This was however recently ruled out by Winn et al. (2017) based on the different metallicity distribution of the host stars.

The second hypothesis for this dearth of short-period EGP is that the distance at which planets stop their migration depends on the planet mass. This would be the 
case if the magnetospheric cavity in the inner-edge of protoplanetary disks, which is supposed to stop hot Jupiters migration (Chang et al. 2010), depends on the mass of the disk and indirectly to the mass of the planet (Mazeh et al.|2016). This would also be the case if hot Jupiters migrated through a high-eccentricity and tidal circularisation, as the minimum distance between planets and their star depends on their Roche limit, which also depends on their mass (Matsakos and Königl 2016). However, there is evidence against this high-eccentricity migration model for hot Jupiters, as presented in Schlaufman and Winn (2016). Therefore, this dearth of planets is currently not clearly understood.

\section{Multiplicity of EGP}

Spectroscopic surveys of hot Jupiters revealed that about half of them have longperiod (at several $\mathrm{AU}$ ) massive companions, within the planetary or stellar regime (e.g. Knutson et al. 2014, Neveu-VanMalle et al.|2016). Except in the unique case of the WASP-47 system (Becker et al. 2015) where a hot Jupiter is sandwiched by two low-mass planets, this EGP population does not have nearby low-mass planets. They might have low-mass companions at wide separation but current instrumentation can not detect them. This supports the idea that hot Jupiters could have migrated with two main mechanisms: (1) through interaction with the disk (type I or II migration) or (2) by planet-planet interactions which would make a high eccentricity for the hot-jupiter progenitor caused either by the Lidov - Kozai effect or planet-planet scattering, and before a tidal circularisation (see Ford 2014, for a review).

On the other hand, some temperate and cold giants as well as EGPs in the period valley have inner, low-mass planetary companions (see Fabrycky et al.|2014). This is evidence that these planets had a smooth disk migration that preserved the inner planets, unlike the hot Jupiters. As aforementioned, in some circumstances, the presence of the companion could even be the reason for these EGPs to stay cool and prevent them from migrating inwards and becoming hot Jupiters. The upcoming next-generation instruments, like ESPRESSO (Pepe et al.|2014), will be able to further probe the architecture of EGP systems, and reveal their migration mechanism.

\section{Radius of EGP}

The radius of EGPs that transit in front of their host star can be measured with relatively high precision. The Fig. 2 show all EGP with measured radius as a function of their incident flux. Because the transit method is more sensitive to planet close to their star, most transiting EGPs known to date are highly irradiated. The EGPs receiving more than $10^{9} \mathrm{erg} . \mathrm{cm}^{-1} . \mathrm{s}^{-1}$ exhibit a radius up to $2.2 \mathrm{R}_{4}$ (hence, $25 \mathrm{R}_{\oplus}$ ). With current models of giant planet atmospheres and internal structure (that are calibrated on Jupiter and Saturn), it is not possible to explain such large radius for a 
gaseous planet, unless they are extremely young (Almenara et al.|2015). Several hypothesis are proposed (Baraffe et al. 2014) but they are still debated. Planets receiving an insolation flux of less than $10^{8} \mathrm{erg} . \mathrm{cm}^{-2} \cdot \mathrm{s}^{-1}$ are yet poorly explored. They have orbital period typically longer than a month and require long-duration spacebased photometric surveys, like CoRoT and Kepler to be detected. Nevertheless, the relatively few objects that were detected to date in this low-incident flux regime do not show sign of inflation. Their radii are in the range $0.5-1.2 \mathrm{R}_{4}$ (equivalent to about $6-13 \mathbf{R}_{\oplus}$ ).

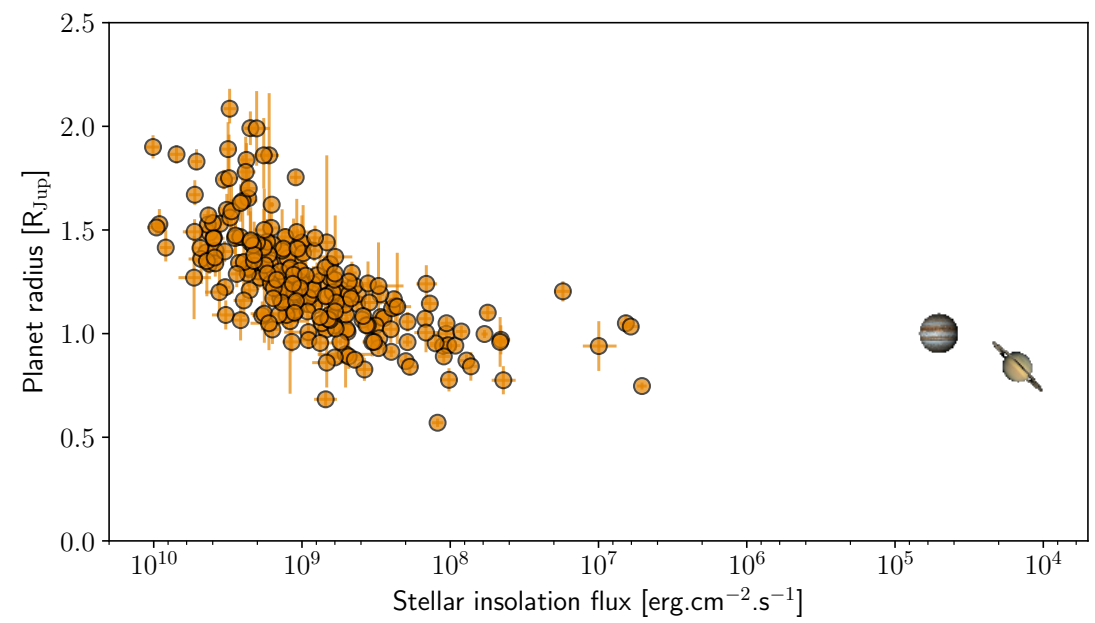

Fig. 2 Radius of EGP as function of their stellar insolation flux. Jupiter and Saturn are displayed for comparison.

As a summary, extrasolar giant planets are objects more massive than about $0.1-0.2 \mathbf{M}_{2}$. The upper-limit in mass is a few tens of $\mathbf{M}_{4}$ but is unclear as the distinction between giant planets and brown dwarfs is still debated. In this regime of planetary mass resides two main populations of planets: the hot Jupiters, with orbital separation of less than 0.1 AU (about 10 days of orbital period) and the temperate/cold giants with semi-major axis greater than about $0.4 \mathrm{AU}$ (about 100 days of period). In the transition, the so-called period valley, relatively few planets have been found. The RV and transit detections have also unveil a clear desert of short-period low-mass giants, where no planet has been found. This desert might be the result of a dramatic evolution or a migration of giant exoplanets. Finally, most of the hot Jupiters only have wide-separation more massive companions while warm to cold giants might have inner, low-mass planetary companions, as detected with current instrumentation. 


\section{Occurrence Rates of EGPs in Different Stellar Populations}

Transit and RV surveys with well defined and characterised stellar samples can be used to derive the occurrence rates of EGPs. One of the main challenges to derive occurrence rate is to correctly estimate the bias inherent of each technique or survey. In the case of the transit surveys, which have targeted relatively faint stars, another challenge is to characterise precisely the observed stellar sample (see Huber et al. 2014; Damiani et al.2016, in the case of Kepler and CoRoT, respectively).

Occurrence rates of EGPs have been derived based on different surveys that observed different stellar populations across the Galaxy. The values are reported in Table 1 and discussed below. The most up-to-date value of each survey is also displayed in Fig. 3 for comparison. For clarity of the plot, only values with a relative precision better than $50 \%$ are shown.

\section{The Solar Neighbourhood}

For now, only RV experiments substantially surveyed the solar neighbourhood and were used to determine the occurrence rates of EGPs. The two main surveys of exoplanets in RV are the Californian Planet Search (CPS) and the Geneva-lead survey, initiated by Marcy et al. and Mayor et al., respectively.

The CPS survey mostly used the Keck and Lick telescopes with their respective high-resolution iodine-cell based spectrographs. Some observations were also performed with the Anglo-Australian Telescope. The occurrence rate estimates were first published in Marcy et al. (2005), and subsequently in Cumming et al. (2008), Howard et al. (2010), and Wright et al. (2012). The various studies used different sample selections. The latter and most up-to-date study probed the solar neighbourhood with a magnitude-limited sample $(\mathrm{V}<8)$ focused on FGK dwarfs. In this sample, 836 stars were observed with a least 5 epochs which is sufficient to detect EGPs with short orbital periods. They defined hot Jupiters as EGPs with periods of less than 10 days and more massive than mass $0.1 \mathrm{M}_{4}$. Ten hot Jupiters were found in their sample, which corresponds to an occurrence rate of $1.2 \pm 0.38 \%$ (see Table 1 . No occurrence rate was specifically derived for the valley period and temperate/cold giant populations, based on the CPS survey. Howard et al. (2010) reported six planets more massive than $30 \mathrm{M}_{\oplus}$ with orbital periods less than 50 days found in a sub-sample of 122 FGK dwarfs, leading to an occurrence rate in this domain of $4.9 \pm 2.0 \%$.

The Geneva-lead survey used both the CORALIE and HARPS spectrographs. The surveys is a volume-limited sample of southern stars up to 50pc focusing on low-activity FGK dwarfs. It is fully described in Mayor et al. (2011) and in Sousa et al. (2011). In total 822 stars were observed by at least one instrument. A total of 155 planets and 6 candidates were detected in this sample, among which 89 have a minimum mass above $50 \mathrm{M}_{\oplus}$ and are considered as EGP. Correcting for the survey 
completeness, it gives an overall occurrence rate of EGP within 10 years at the level of $13.9 \pm 1.7 \%$ (Mayor et al. 2011). Selecting only hot Jupiters, valley-period giants, and temperate giants (within 400 days to allow comparison with the Kepler transit survey), it gives occurrence rates of $0.83 \pm 0.34 \%, 1.64 \pm 0.55 \%$, and $2.90 \pm 0.72 \%$ as derived in Santerne et al. (2016), see also Table 1. Note that a first attempt to estimate the occurrence rate of EGP in the solar neighbourhood was done with the ELODIE (northern) spectrograph, leading to a low-precision value of $0.7 \pm 0.5 \%$ for hot Jupiters (Naef et al.2005).

In the solar neighbourhood, the occurrence rate of hot Jupiters has been measured by two slightly different surveys at the level of about $1 \%$.

\section{The Kepler Field}

The Kepler space telescope surveyed about 200000 stars during four years (Borucki et al.2009). The field of view was the same during the prime mission. This field is located about 10 degrees above the galactic plane. Most of the FGK dwarf targets observed in this field are located at several hundreds of parsec away, up to a few kiloparsec.

Nearly 4700 planet candidates were detected (Coughlin et al. 2016), among which more than 2200 are confirmed or validated (e.g. Rowe et al. 2014; Morton et al. 2016). Therefore, this photometric surveys is a gold mine for statistical analysis, which was actually the prime objective of the mission (e.g. Batalha|2014). Statistical properties of exoplanets, including of EGPs, were already derived with the first month (out of 48 in total) of data only (Borucki et al. 2011). It was subsequently revised and improved as more data were collected and more candidates were detected. Occurrence rates of exoplanets based on the Kepler detections were discussed in numerous papers (see https://exoplanetarchive.ipac.caltech.edu/ docs/occurrence_rate_papers.html for an updated list). In the particular case of EGPs, the occurrence rate was first derived in (Howard et al.2012) for planets up to $0.25 \mathrm{AU}$ based on the first four months of data. In this paper, the occurrence rate of hot Jupiters (defined as planets with a radius in the range $8-32 R_{\oplus}$ and orbital period of less than 10 days) was estimated to $0.4 \pm 0.1 \%$. However, because eclipsing low-mass stellar companion can perfectly mimic the transit of an EGP (the so-called false positives Santerne et al. 2012, e.g.), the reliability of the transit detections to be bona-fide exoplanets has to be taken into account. Correcting this bias using spectroscopic ground-based observations with the SOPHIE instrument, Santerne et al. (2012) re-evaluated the occurrence rate of hot Jupiters (defined as planets with a transit depth in the range $0.4 \%-3 \%$ and orbital periods less than 10 days) in the Kepler field to be $0.57 \pm 0.07 \%$ also based on the first 4 months of Kepler data. Although the two values are fully consistent, the main difference between these two 
studies is the selection criteria for hot Jupiters and the fact that in the latter analysis, all false positives were screened out with ground-based spectroscopy.

The false-positive rate of Kepler EGPs turned out to be significantly higher (Santerne et al.2012, at the level of 35\% for EGP within 25 days detected based on the first 16 months of Kepler data; ) than previously estimated (at the level of 5\% Morton and Johnson 2011). Therefore, to derive more reliable occurrence rates, Fressin et al. (2013) simulated the population of false positives, compared it with the population of planetary candidates, and derived occurrence rates for all populations of planets detected in the Kepler field, up to about 400 days of orbital periods. For EGP, considered as planets with a radius in the range $6-22 \mathrm{R}_{\oplus}$, they reported occurrence rates of $0.43 \pm 0.05 \%, 1.56 \pm 0.11 \%$, and $3.24 \pm 0.25 \%$ for the hot Jupiter, period-valley, and temperate EGPs, respectively (see Table 1 .

Extending the sample of Santerne et al. (2012) to include all EGP up to 400 days of period and detected in the four years of the prime mission (from DR24), Santerne et al. (2016) observed 125 EGP candidates with the SOPHIE spectrograph to screen out impostors. They finally reported a false-positive rate at the level of $55 \%$ over the whole sample and derived occurrence rates, cleaned from false positives, of $0.47 \pm 0.08 \%, 0.90 \pm 0.24 \%$, and $3.19 \pm 0.73 \%$ for the hot jupiter, period-valley, and temperate giant populations. These values are fully consistents with those derived by Fressin et al. (2013), except for the period-valley EGP where the false-positive contamination was underestimated in the latter study.

In the Kepler field, the occurrence rate of hot Jupiters has been estimated by different teams using different approaches to correct for the false positives. The most up-to-date value is $0.47 \pm 0.08 \%$, which is about half the one reported in the solar neighborhood.

\section{The CoRoT eyes}

The french-led CoRoT space mission (Baglin et al. 2006) was the first space mission performing a photometric survey of stars to search for transiting exoplanets. The satellite was pointing different fields of view continuously for periods up to 150 days in two directions (the so-called CoRoT eyes): towards the galactic center and galactic anti-center. The stellar samples observed by CoRoT were located at several hundreds of parsec, up to a few kiloparsec near the Galactic plane. As a consequence, the mission was probing two different stellar populations, both being different from the solar neightborhood and the Kepler field.

CoRoT was initially designed to explore and characterise the populations of small and long-period planets that are out of reach from ground-based surveys. Deriving the occurrence rates of planets with precision requires to understand the various bias introduced by the different detection teams across Europe (Moutou et al. 2005) as well as the photometric and spectroscopic follow-up process. Moreover, the stellar 
populations were poorly characterised, hence limiting the precision of occurrence rates. As a consequence, compared to Kepler, relatively few occurrence rate studies have been attempted.

Guenther et al. (2012) performed a massive spectroscopic survey of CoRoT targets in a few pointings toward the galactic anti-center to better characterise the stellar population and derived a first estimate of the occurrence of hot jupiters of $0.4 \pm 0.2 \%$. This value was later updated by Moutou et al. (2013) to $1.0 \pm 0.3 \%$ to include more detections from both galactic directions.

Re-analysing the 5.5 years of the CoRoT/Exoplanet programme with an homogeneous transit detection pipeline and selecting the candidates to minimise the bias introduced by the follow-up observations, Deleuil et al. (2018) derived final values for the occurrence rates of hot jupiters in each of the CoRoT eyes. They reported values of $0.95 \pm 0.26 \%$ and $1.12 \pm 0.31 \%$ for the center and anticenter fields, respectively. For EGP with orbital period in the range $10-100$ days, the rate is estimated, based on relatively few detections, to $1.53 \pm 1.1 \%$.

In both of the two CoRoT eyes, the occurrence rate of hot Jupiters has been estimated to be at the level of about $1 \%$, in agreement with the value observed in the solar neighborhood.

\section{Ground-based transit surveys}

Many photometric surveys have been performed from the ground with the sensitivity to detect hot Jupiters in many different stellar populations. However, photometric data are highly heterogenous because of variable sky conditions, hence strongly limiting the accurate determination of the survey completeness. The bias introduced by the candidate selection and the follow-up are also challenging to estimate. This explains why the large photometric ground-based surveys, targeting the whole sky, like SuperWASP (Pollacco et al. 2006) and HATNet (Bakos et al. 2004), have not derived yet occurrence rates. Although this would be extremely interesting, this represents a titanic work.

Relatively modest ground-based photometric surveys have been performed on very specific fields of view, allowing to derive estimates of the occurrence rates of the shortest period giant planets. This is the case of the following surveys:

- The OGLE survey that observed stars in Carina and the galactic bulge (Gould et al. 2006). They derived the occurrence rate of EGPs within $1-3$ days and $3-$ 5 days of orbital period. Computing the value for the period range $1-5$ days and assuming pure Poisson noise, the occurrence rate is $0.88 \pm 0.39 \%$.

- The SWEEPS survey (Sahu et al.2006) targeted the galactic bulge and reported an occurrence rate of EGP within 4.2 days of $0.4_{-0.2}^{+0.4} \%$. 
- The Deep MMT survey targeted a field of view in the vicinity of the open cluster M37 (Hartman et al. 2009). They reported a 95\% confidence upper-limit on the occurrence rate of EGP within 5 days of orbital period and larger than $1 \mathrm{R}_{4}$ at the level of $3.2 \%$ and $8.3 \%$ for field and cluster member stars.

- The SuperLupus survey targeted stars in the Lupus constellation and reported an occurrence rate of hot jupiters at the level of $0.1_{-0.08}^{+0.27} \%$ Bayliss and Sackett 2011).

These four surveys were targeting relatively faint stars, most of them being fainter than Rmag $\sim 15$ and up to 23 . The characterisation of the system with spectroscopic mean requires large telescopes for such faint stars and is thus limited. The number of candidates detected by these surveys is extremely low. This is even lower when considering bona-fide EGPs (up to 5 EPGs for the OGLE survey). These occurrence rates are therefore based on small-number statistics and should be interpreted with cautious.

\section{In Open Clusters}

Clusters are pristine targets to test formation and evolution scenarios of exoplanets in different environments than field stars. They also have a well constrained age and metallicity. They have been surveyed for more than a decade with no detection (e.g. Gilliland et al. 2000, Hartman et al. 2009). Only recently, the first EGPs have been detected in clusters (Quinn et al. 2012). Based on three detections in radial velocity out of 66 stars in the open cluster M67, Brucalassi et al. (2016) reported an occurrence rate of hot Jupiter at the level of $5.6_{-2.6}^{+5.4} \%$ and $4.5_{-2.5}^{+4.5} \%$ for the single stars and the full sample, respectively. Although these values are much higher than any other estimate, they are also based on very small number statistics, as reflected by their large uncertainties. They should also be interpreted with cautious.

\section{The Brown-Dwarf Desert}

Brown dwarfs are sub-stellar objects with a mass greater than about $10-20 \mathrm{M}_{4}$, although this is still highly debated (Chabrier et al.2014), and up to about $80 \mathbf{M}_{4}$. These objects are extremely rare in orbit around a solar-type stars within a few AU. This is the so-called brown-dwarf desert (e.g. Marcy and Butler 2000). Based on radial velocity survey, the occurrence rate of brown dwarfs within $3 \mathrm{AU}$ has been estimated to be as low as $0.1_{-0.1}^{+0.2} \%$ (Grether and Lineweaver 2006). Using the $\mathrm{Ke}$ pler data and the SOPHIE spectrograph, Santerne et al. (2016) slightly improved this value to $0.29 \pm 0.17 \%$ for brown dwarfs companion of FGK stars with an orbital period of less than 400 days. This value is consistent with the one found by Csizmadia et al. (2015) of $\sim 0.2 \%$, for objects within 10 days of orbital period, based on the CoRoT detections. Although these estimates are based on small number statistics 
they reveal that the occurrence rate of companion brown dwarfs in the desert is 5 - 15 times lower than for EGP in the same stellar sample (Csizmadia et al. 2015, Santerne et al.|2016.

\section{Comparison of the Occurrence Rates}

With two decades of exploration of EGPs, their occurrence rate has been measured by various spectroscopic and photometric surveys in different stellar environments, from the solar neighbourhood, to open clusters. If significant differences are found in these rates between different stellar fields, it will give important insights on the impact of the stellar properties to the formation of EGPs. Comparing the occurrence rates of hot Jupiters in the various surveys aforementioned, it is remarkable that all but the Kepler field reported a mean value of $1.00 \pm 0.14 \%$. In the Kepler field, the observed value is nearly half, of $0.47 \pm 0.08 \%$. Under the assumption that the occurrence rate of hot Jupiter is the same in the solar neighbourhood, the OGLE survey and in both of the CoRoT eyes, then the difference with the Kepler field is at more than $3-\sigma$.

While the Kepler field was initially thought to be of lower metallicity (see next section for the impact of the stellar metallicity of the EGP formation) compared to the other surveys (Santerne et al. 2016, and references therein), massive spectroscopic survey of Kepler stars reported it to be consistent with a solar metallicity and furthermore, compatible with the other surveys (Dong et al. 2014; Guo et al. 2017). This difference in the rates is therefore unlikely explained by the difference of metallicity of the stellar field. Several other solutions are proposed such as a

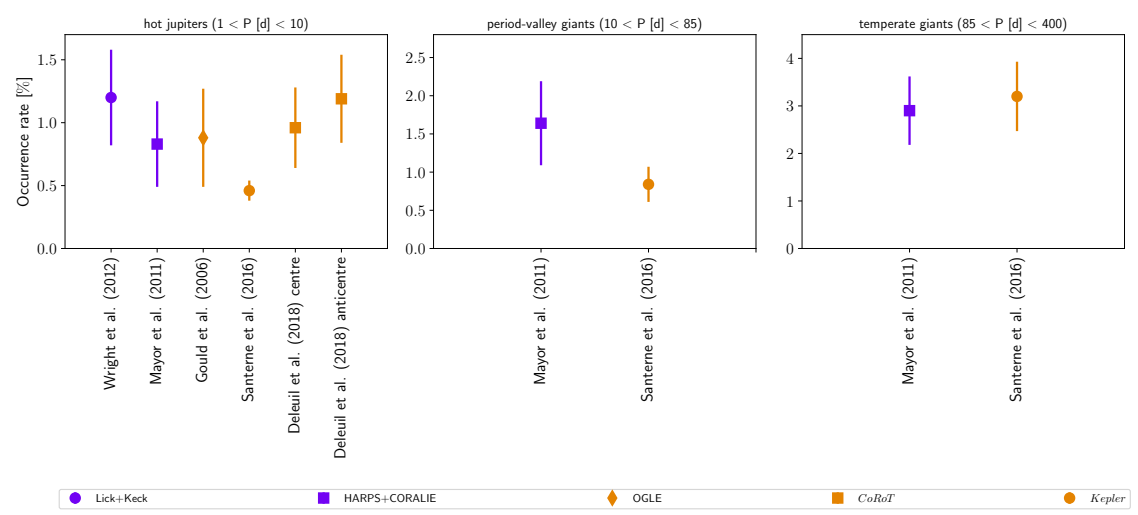

Fig. 3 Occurrence rates of the different populations of EGP (left: hot Jupiter ; middle: periodvalley giants ; right: temperate giants) by different surveys. Violet marks are for RV surveys and orange marks are for photometric surveys. Note the different scale between the three panels. Adapted from Santerne et al. (2016). 
different stellar multiplicity rate or some significant bias in the stellar population observed by Kepler, but it remains unclear today.

It is interesting to note that while the occurrence rate of hot Jupiter is significantly lower in the Kepler field compared to other surveys, this does not seem to be the case for temperate EGPs (see Fig. 3). The occurrence rates of EGPs in the period valley and of temperate planets, assumed to be within orbital periods of $10 \mathrm{~d}-85 \mathrm{~d}$ and $85 \mathrm{~d}-400 \mathrm{~d}$ (respectively) have been estimated in both the solar neighbourhood based on the HARPS+CORALIE sample and in the Kepler field (Mayor et al.2011, Santerne et al. 2016). Although the uncertainties are large, the occurrence rates of giant planets at long orbital period are very consistent between the two surveys. For the transition population, if the depletion of EGPs is real, it is not significant in the data. Further investigation are mandatory to fully understand this picture.

\section{Relation with Host Star Properties}

The environmental conditions in which EGPs form should influence their physical properties that are observed now. To test what are the key ingredients for planet formation, one can use the properties of the host stars as proxy of the initial conditions. The relation between the host star and the EGP properties provides extremely important constraints to understand their formation. The main stellar properties that have been reported so far to significantly influence the formation of EGPs are the metallicity and the mass.

\section{The Stellar Metallicity}

A connection between the stellar metallicity and the presence of an EGP was first identified by Gonzalez (1997) based on the first four EGPs discovered. They were found to orbit preferentially metal-rich stars. The correlation between the presence of an EGP and the metallicity of the host star was later revised and further characterised as the number of EGPs increased, in particular in Santos et al. (2001, 2003) and in Fischer and Valenti (2005). This correlation was seen as an observational evidence for the core-accretion mechanism as the primary formation scenario for EGP. Indeed, protoplanetary disks accrete materials to form planetesimal more efficiently if they are rich in metals (Pollack et al. 1996) than if they are depleted in metals. On the other hand, the disk instability scenario was predicting a flat distribution of EGP-host metallicities (Boss 2002). However, the latest versions of the disk instability mechanism are capable of reproducing this correlation (Nayakshin and Fletcher|2015; Nayakshin|2017).

Figure 4 displays the cumulative distribution function of the metallicity of all EGP hosts known to date (data from the SweetCat catalog ; Santos et al.|2013), compared to the distribution of metallicity in the solar neighbourhood (Haywood 
Table 1 Occurrence rates of EGP for different ranges of orbital periods from different studies. All values are in percent.

\begin{tabular}{|c|c|c|c|c|}
\hline Reference & $\begin{array}{c}\text { Hot Jupiters } \\
P<10 \mathrm{~d} \\
{[\%]} \\
\end{array}$ & $\begin{array}{c}\text { Period-Valley Giants } \\
10<P<85 \mathrm{~d} \\
{[\%]} \\
\end{array}$ & $\begin{array}{c}\text { Temperate Giants } \\
85<P<400 \mathrm{~d} \\
{[\%]}\end{array}$ & Survey / Field \\
\hline Marcy et al. $(2005)$ & $1.2 \pm 0.2$ & - & - & Keck+Lick+AAT \\
\hline Naef et al. (2005) & $0.7 \pm 0.5^{(a)}$ & - & - & ELODIE \\
\hline Cumming et al. (2008) & $1.5 \pm 0.6$ & - & - & Keck \\
\hline Mayor et al. (2011) & $0.83 \pm 0.34^{(b)}$ & $1.64 \pm 0.55^{(b)}$ & $2.90 \pm 0.72^{(b)}$ & HARPS+CORALIE \\
\hline Wright et al. (2012) & $1.20 \pm 0.38$ & - & - & Keck+Lick \\
\hline Brucalassi et al. (2016) & $4.5_{-2.5}^{+4.5}$ & - & - & M67 \\
\hline Gould et al. (2006) & $0.88 \pm 0.39^{(c)}$ & - & - & OGLE \\
\hline Sahu et al. $(2006)$ & $0.4_{-0.2}^{+0.4}(d)$ & - & - & SWEEPS \\
\hline Hartman et al. (2009) & $<8.3^{(e)}$ & - & - & Deep MMT (M37) \\
\hline Hartman et al. $(\overline{2009})$ & $<3.2^{(e)}$ & - & - & Deep MMT (field stars) \\
\hline Bayliss and Sackett (2011) & $0.10_{-0.08}^{+0.27}$ & - & - & SuperLupus \\
\hline Howard et al. (2012) & $0.4 \pm 0.1$ & - & - & Kepler \\
\hline Santerne et al. (2012) & $0.57 \pm 0.07$ & - & - & Kepler \\
\hline Fressin et al. 2013 & $0.43 \pm 0.05$ & $1.56 \pm 0.11$ & $3.24 \pm 0.25$ & Kepler \\
\hline Moutou et al. 2013, & $1.0 \pm 0.3^{(f)}$ & - & - & All CoRoT fields \\
\hline Santerne et al. (2016) & $0.47 \pm 0.08$ & $0.90 \pm 0.24$ & $3.19 \pm 0.73$ & Kepler \\
\hline Deleuil et al. $(2018)$ & $0.95 \pm 0.26$ & & - & CoRoT center fields \\
\hline Deleuil et al. $(\overline{2018})$ & $1.12 \pm 0.31$ & $53 \pm 1.1$ & - & CoRoT anti-center fields \\
\hline
\end{tabular}

The horizontal line separates the values determined by RV surveys (above) from the ones determined by photometric transit surveys (below).

(a) Only for periods less than 5 days, but no planet is detected in the sample stars with orbital periods between 5 and 10 days. Therefore, a different period bin, up to 10 days would have provided the same value.

(b) Values derived in Santerne et al. (2016) based on the detections and bias correction provided in Mayor et al. (2011). The occurrence rate of hot Jupiters slightly differ from the original paper to account only for planets within 10 days of period. The only difference is the planet HD108147b $(\mathrm{P}=10.89 \mathrm{~d})$ that is included here in the population of the period-valley giants and not in the hot Jupiter population.

(c) Based on detections up to 5 days. Uncertainty estimated from pure Poisson noise.

(d) Based on detections up to 4.2 days.

(e) $95 \%$ confidence upper-limit for $1 \mathrm{R}_{4}$ planets with orbital periods up to 5 days.

(f) Update of the value provided in Guenther et al. (2012) based on the first few CoRoT fields.

2001). While about $50 \%$ of stars in the Sun's vicinity are metal-rich (relative to the Sun value), nearly $80 \%$ of EGP hosts are metal rich. This clearly illustrates that EGP prefers to form around metal-rich stars.

When the number of EGPs was high enough, this correlation was characterised to be reproduced by a power law of the form:

$$
\mathscr{P}(\text { planet })=\alpha[\mathrm{Fe} / \mathrm{H}]^{\beta},
$$

with $\alpha=0.03$ and $\beta=2.0$ (Fischer and Valenti 2005). This functional form was then revisited in Johnson et al. (2010) and Mortier et al. (2013) to account for the 
role of the stellar mass (see next section) and discuss the shape of the distribution at low-metallicity values. The number of EGPs known today is still too low to precisely constrain the form of the correlation in the low metallicity regime.

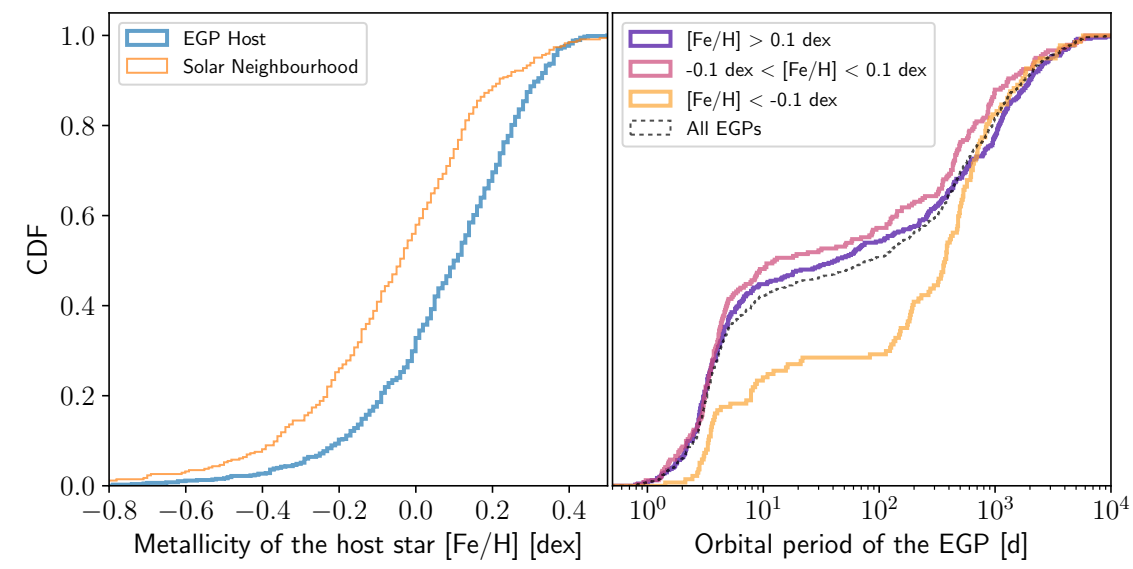

Fig. 4 (Left) Cumulative distribution functions (CDF) of the metallicity of EGP host stars (thick blue line) and of the solar neighbourhood (thin orange line ; Haywood 2001). (Right) CDF of the orbital period of EGPs for three populations of host stars: the metal-poor (orange), the solar metallicity (pink), and metal-rich (violet) stars. The dotted line is the CDF for all EGPs. Only those detected by the transit and RV techniques are displayed here. The metallicity data of the host stars are from the Sweet-Cat catalogue (Santos et al.2013).

The metallicity of the host star does not only impact the presence of planets, it also shape their period distribution. As pointed out by Adibekyan et al. (2013), EGP in metal-poor systems are found at much larger separation than those in the metalrich conterpart. This is illustrated in Fig. 4 where EGPs orbiting stars with $[\mathrm{Fe} / \mathrm{H}]$ $<-0.1$ dex clearly have much longer orbital periods than those orbiting stars more rich in metals. This is explained by the fact EGPs still form around metal-poor stars, but slower than in metal-rich system. As a consequence, their migration is also less efficient and they are observed close to their formation place.

\section{The Stellar Mass}

The mass of the central star is known to impact the formation of EGPs. Indeed, the mass of the protoplanetary disk scale with the mass of the star. Hence, the more massive disks have the potential to form more efficiently massive planets, compared to low-mass disks. On one end, extremely few giant planets have been found so far to orbit the low-mass M dwarfs (among the few cases, there are GL $876 \mathrm{~b}$ and 
Kepler-42 b ; Delfosse et al. 1998, Johnson et al. 2012, respectively). Therefore, protoplanetary disks surrounding $\mathrm{M}$ dwarfs are not massive enough to form efficiently EGPs.

On the other end, massive stars form with massive disks and should form efficiently EGPs. This is however challenging to explore as $\mathrm{O}, \mathrm{B}, \mathrm{A}$, and early $\mathrm{F}$ stars have few lines in the optical and rotate fast hence strongly limiting the radial velocity technique. The approach employed by e.g. Johnson et al. (2007) was to search for EGPs orbiting retired A stars, i.e. giant stars for which precise RVs can be obtained. While a relatively large number of EGPs were found around evolved stars, determining the mass of the host star accurately is challenging without the use of asteroseismology. Indeed stellar evolutionary tracks from a wide range of stellar mass converge towards the so-called giant branch. The mass determination of the retired A stars, then used to quantify the impact of the stellar mass on the formation of EGP (Johnson et al. 2010), is controversial (Lloyd 2011, 2013). Nevertheless, EGPs are found more abundant around $\mathrm{K}$ giants, than around solar-like stars (Bowler et al. 2010), hence confirming the trends observed with $\mathrm{M}$ dwarfs. It is however expected that a cut-off high mass exists, around which EGP do not form, e.g. because the star evolves too fast off of the main-sequence, or because of intense stellar winds.

The properties of the central star have a direct impact of the formation of EGP. The most important is the host metallicity. EGPs form preferentially around metal-rich stars. Those forming around metal-poor stars have a wider separation than their metal-rich counterparts. The mass of the central star also play an important role on the formation of EGP. The more massive the host star is, the more likely they form EGP. As a consequence, EGPs are very rare around $\mathrm{M}$ dwarfs.

\section{Conclusions}

Extrasolar giant planets have been explored for more than two decades with transit and radial velocity surveys, leading to several hundreds of detections. From this large sample of objects, it is possible to infer about their physical properties and to identify different populations. Both are providing important constraints for planet formation and migration theories. The EGPs have been found to have a mass greater than about $0.1-0.2 \mathrm{M}_{4}$, which corresponds to the critical mass to start runaway accretion and type II migration. The upper-mass limit of EGPs is unclear and still debated. It would be located between $13 \mathrm{M}_{4}$, the official limit as defined by the IAU, up to $25 \mathrm{M}_{4}$. The EGPs that received a moderate stellar irradiation exhibit a radius in the range $0.5-1.2 \mathrm{R}_{4}$ while those extremely close to their star might have radius up to about $2.2 \mathrm{R}_{4}$.

Among these EGPs detected by transit and RV surveys, three populations can be identified: 
1. Very close to the host stars (with an orbital period of less than about 10 days) exists a population of EGPs called the hot Jupiters. These planets have an occurrence rate at the level of about $1 \%$ as observed in the solar neighborhood with the RV surveys (HARPS+CORALIE and Keck+Lick among the main surveys), in both of the CoRoT eyes as well as in the OGLE photometric survey. However, this value has been reported to be nearly half in the Kepler field for a reason that is still unclear. The formation and migration mechanisms for these EGPs is still debated. Among the most probable scenarios, there is the high-eccentricity with tidal circulation scenario caused by the Lidov - Kozai mechanism or planetplanet scattering. Another scenario would be a disk-driven migration, although this hypothesis is not clearly compatible with the diverse orbital obliquities of these planets.

2. Much farther out from the host star (with an orbital period greater than about 100 days) exists a population of cool (or temperate) EGPs. These planets have been mainly explored by the RV surveys as their transit probability is extremely low (less than about 1\%) and require long-duration, high-cadence space-based observations like with the CoRoT or Kepler missions. Their occurrence rate can reach up to about $14 \%$ for EGPs within 10 years of orbital periods. In the period range $85-400$ d, covered by both the RV and Kepler surveys, their occurrence rate has been estimated at the level of $3 \%$. This value is fully consistent between the two surveys, albeit the uncertainties are relatively large. These EGPs likely formed in-situ or had a relatively soft migration from their birth place.

3. In between the hot jupiter and cool/temperate giants resides a transition population of planets. They are called the period-valley giants or even warm giants. They have an orbital period roughly in the range 10-100 days. Their occurrence rate has been reported up to $1.6 \%$ in the solar neighbourhood and down to $0.9 \%$ in the Kepler field, albeit the uncertainties are large enough to prevent this difference to be statistically significant. The origin of these EGPs is still unclear and they are probably in the tail of the distribution of the other two main populations.

The properties of the host star, proxy of the properties of the protoplanetary disk, has been found to have an impact on the formation of EGPs. The more metal-rich stars are, the more likely they form EGPs. The metal-poor stars still form EGPs but these planets are observed with a much wider orbital separation than those orbiting metal-rich stars. This is explained by the fact the core-accretion mechanism is less efficient to form giant planets in metal-poor disks. Therefore, when EGPs form in metal-poor disk, they need more time to accrete materials and hence migrate less efficiently. The mass of the star also impacts the formation of EGPs: the more massive the central star is, the more likely they formed with EGPs. As a consequence, EGPs are extremely rare in orbit around the low-mass M dwarfs.

More than two decades after the discovery of the first EGP, and at a time when the community interest moves towards small and cool planets, there are still many open questions to explain all the observed properties of the EGPs. For instance, the formation and migration scenario of hot jupiters, the transition between EGPs and brown dwarfs, the reason for the inflated radius of highly-irradiated EGPs, the difference between the occurrence rate of hot Jupiters as observed in the Kepler 
field and the one of all the other surveys, all these questions are still debated. To solve them, more theoretical models have to be developed together with stronger observational constraints.

\section{Cross-References}

- Radial Velocities as an Exoplanet Discovery Method

- Transit Photometry as an Exoplanet Discovery Method

- High-Precision Spectrographs for Exoplanet Research: Elodie, Coralie, Sophie and HARPS

- Space Missions for Exoplanet Science with CoRoT

- Space Missions for Exoplanet Science: Kepler / K2

- The SuperWASP and NGTS Exoplanet Surveys

- The HATNet and HATPI Exoplanet Surveys

- Mass-Radius Relations of Giant Planets: The Radius Anomaly and Interior Models

- Exoplanet Occurrence Rates from Transit and RV Surveys

- Formation of Giant Planets

- Extrasolar Planets Population Synthesis

\section{References}

Adibekyan VZ, Figueira P, Santos NC et al. (2013) Orbital and physical properties of planets and their hosts: new insights on planet formation and evolution. A\&A560:A51

Akeson RL, Chen X, Ciardi D et al. (2013) The NASA Exoplanet Archive: Data and Tools for Exoplanet Research. PASP125:989

Almenara JM, Damiani C, Bouchy F et al. (2015) SOPHIE velocimetry of Kepler transit candidates. XV. KOI-614b, KOI-206b, and KOI-680b: a massive warm Jupiter orbiting a G0 metallic dwarf and two highly inflated planets with a distant companion around evolved F-type stars. A\&A575:A71

Baglin A, Auvergne M, Boisnard L et al. (2006) CoRoT: a high precision photometer for stellar ecolution and exoplanet finding. In: 36th COSPAR Scientific Assembly, COSPAR Meeting, vol 36

Bakos G, Noyes RW, Kovács G et al. (2004) Wide-Field Millimagnitude Photometry with the HAT: A Tool for Extrasolar Planet Detection. PASP116:266-277

Baraffe I, Chabrier G, Fortney J Sotin C (2014) Planetary Internal Structures. Protostars and Planets VI pp 763-786

Batalha NM (2014) Exploring exoplanet populations with NASA's Kepler Mission. Proceedings of the National Academy of Science 111:12,647-12,654

Bayliss DDR Sackett PD (2011) The Frequency of Hot Jupiters in the Galaxy: Results from the SuperLupus Survey. ApJ 743:103

Becker JC, Vanderburg A, Adams FC, Rappaport SA Schwengeler HM (2015) WASP-47: A Hot Jupiter System with Two Additional Planets Discovered by K2. ApJ812:L18 
Borucki W, Koch D, Batalha N et al. (2009) KEPLER: Search for Earth-Size Planets in the Habitable Zone. In: Pont F, Sasselov D Holman MJ (eds) Transiting Planets, IAU Symposium, vol 253, pp 289-299, DOI 10.1017/S1743921308026513

Borucki WJ, Koch DG, Basri G et al. (2011) Characteristics of Kepler Planetary Candidates Based on the First Data Set. ApJ728:117

Boss AP (2002) Stellar Metallicity and the Formation of Extrasolar Gas Giant Planets. ApJ567:L149-L153

Bowler BP, Johnson JA, Marcy GW et al. (2010) Retired A Stars and Their Companions. III. Comparing the Mass-Period Distributions of Planets Around A-Type Stars and Sun-Like Stars. ApJ709:396-410

Brucalassi A, Pasquini L, Saglia R et al. (2016) Search for giant planets in M67. III. Excess of hot Jupiters in dense open clusters. A\&A592:L1

Bruno G, Almenara JM, Barros SCC et al. (2015) SOPHIE velocimetry of Kepler transit candidates. XIV. A joint photometric, spectroscopic, and dynamical analysis of the Kepler-117 system. A\&A573:A124

Chabrier G, Johansen A, Janson M Rafikov R (2014) Giant Planet and Brown Dwarf Formation. Protostars and Planets VI pp 619-642

Chang SH, Gu PG Bodenheimer PH (2010) Tidal and Magnetic Interactions Between a Hot Jupiter and its Host Star in the Magnetospheric Cavity of a Protoplanetary Disk. ApJ708:1692-1702

Coughlin JL, Mullally F, Thompson SE et al. (2016) Planetary Candidates Observed by Kepler. VII. The First Fully Uniform Catalog Based on the Entire 48-month Data Set (Q1-Q17 DR24). ApJS224:12

Crida A Bitsch B (2017) Runaway gas accretion and gap opening versus type I migration. Icarus 285:145-154

Csizmadia S, Hatzes A, Gandolfi D et al. (2015) Transiting exoplanets from the CoRoT space mission. XXVIII. CoRoT-33b, an object in the brown dwarf desert with 2:3 commensurability with its host star. A\&A584:A13

Cumming A, Butler RP, Marcy GW et al. (2008) The Keck Planet Search: Detectability and the Minimum Mass and Orbital Period Distribution of Extrasolar Planets. PASP120:531

Damiani C, Meunier JC, Moutou C et al. (2016) Stellar classification of CoRoT targets. A\&A595:A95

Deleuil M, Aigrain S, Moutou C et al. (2018) Planets, candidates, and binaries from the CoRoT/Exoplanet programme: the CoRoT transit catalogue. A\&A, in press

Delfosse X, Forveille T, Mayor M et al. (1998) The closest extrasolar planet. A giant planet around the M4 dwarf GL 876. A\&A338:L67-L70

Dong S, Zheng Z, Zhu Z et al. (2014) On the Metallicities of Kepler Stars. ApJ789:L3

Fabrycky DC, Lissauer JJ, Ragozzine D et al. (2014) Architecture of Kepler's Multi-transiting Systems. II. New Investigations with Twice as Many Candidates. ApJ790:146

Fischer DA Valenti J (2005) The Planet-Metallicity Correlation. ApJ622:1102-1117

Ford EB (2014) Architectures of planetary systems and implications for their formation. Proceedings of the National Academy of Science 111:12,616-12,621

Fressin F, Torres G, Charbonneau D et al. (2013) The False Positive Rate of Kepler and the Occurrence of Planets. ApJ766:81

Gilliland RL, Brown TM, Guhathakurta P et al. (2000) A Lack of Planets in 47 Tucanae from a Hubble Space Telescope Search. ApJ545:L47-L51

Gonzalez G (1997) The stellar metallicity-giant planet connection. MNRAS285:403-412

Gould A, Dorsher S, Gaudi BS Udalski A (2006) Frequency of Hot Jupiters and Very Hot Jupiters from the OGLE-III Transit Surveys toward the Galactic Bulge and Carina. Acta Astron56:1-50

Grether D Lineweaver CH (2006) How Dry is the Brown Dwarf Desert? Quantifying the Relative Number of Planets, Brown Dwarfs, and Stellar Companions around Nearby Sun-like Stars. ApJ640:1051-1062

Guenther EW, Gandolfi D, Sebastian D et al. (2012) Multi-object spectroscopy of stars in the CoRoT fields. II. The stellar population of the CoRoT fields IRa01, LRa01, LRa02, and LRa06. A\&A543:A125 
Guo X, Johnson JA, Mann AW et al. (2017) The Metallicity Distribution and Hot Jupiter Rate of the Kepler Field: Hectochelle High-resolution Spectroscopy for 776 Kepler Target Stars. ApJ838:25

Hartman JD, Gaudi BS, Holman MJ et al. (2009) Deep MMT Transit Survey of the Open Cluster M37 IV: Limit on the Fraction of Stars with Planets as Small as 0.3R . ApJ695:336-356

Hatzes AP Rauer H (2015) A Definition for Giant Planets Based on the Mass-Density Relationship. ApJ810:L25

Haywood M (2001) A revision of the solar neighbourhood metallicity distribution. MNRAS325:1365-1382

Hébrard G, Arnold L, Forveille T et al. (2016) The SOPHIE search for northern extrasolar planets. $X$. Detection and characterization of giant planets by the dozen. A\&A588:A145

Holman MJ, Fabrycky DC, Ragozzine D et al. (2010) Kepler-9: A System of Multiple Planets Transiting a Sun-Like Star, Confirmed by Timing Variations. Science 330:51

Howard AW, Marcy GW, Johnson JA et al. (2010) The Occurrence and Mass Distribution of Closein Super-Earths, Neptunes, and Jupiters. Science 330:653

Howard AW, Marcy GW, Bryson ST et al. (2012) Planet Occurrence within 0.25 AU of Solar-type Stars from Kepler. ApJS201:15

Huber D, Silva Aguirre V, Matthews JM et al. (2014) Revised Stellar Properties of Kepler Targets for the Quarter 1-16 Transit Detection Run. ApJS211:2

Johnson JA, Fischer DA, Marcy GW et al. (2007) Retired A Stars and Their Companions: Exoplanets Orbiting Three Intermediate-Mass Subgiants. ApJ665:785-793

Johnson JA, Aller KM, Howard AW Crepp JR (2010) Giant Planet Occurrence in the Stellar MassMetallicity Plane. PASP122:905

Johnson JA, Gazak JZ, Apps K et al. (2012) Characterizing the Cool KOIs. II. The M Dwarf KOI254 and Its Hot Jupiter. AJ143:111

Knutson HA, Fulton BJ, Montet BT et al. (2014) Friends of Hot Jupiters. I. A Radial Velocity Search for Massive, Long-period Companions to Close-in Gas Giant Planets. ApJ 785:126

Lloyd JP (2011) "Retired" Planet Hosts: Not So Massive, Maybe Just Portly After Lunch. ApJ739:L49

Lloyd JP (2013) The Mass Distribution of Subgiant Planet Hosts. ApJ774:L2

Marcy G, Butler RP, Fischer D et al. (2005) Observed Properties of Exoplanets: Masses, Orbits, and Metallicities. Progress of Theoretical Physics Supplement 158:24-42

Marcy GW Butler RP (2000) Planets Orbiting Other Suns. PASP112:137-140

Masuda K (2014) Very Low Density Planets around Kepler-51 Revealed with Transit Timing Variations and an Anomaly Similar to a Planet-Planet Eclipse Event. ApJ783:53

Matsakos T Königl A (2016) On the Origin of the Sub-Jovian Desert in the Orbital-periodPlanetary-mass Plane. ApJ820:L8

Mayor M Queloz D (1995) A Jupiter-mass companion to a solar-type star. Nature378:355-359

Mayor M, Marmier M, Lovis C et al. (2011) The HARPS search for southern extra-solar planets XXXIV. Occurrence, mass distribution and orbital properties of super-Earths and Neptunemass planets. ArXiv e-prints

Mazeh T, Holczer T Faigler S (2016) Dearth of short-period Neptunian exoplanets: A desert in period-mass and period-radius planes. A\&A589:A75

Morbidelli A Crida A (2007) The dynamics of Jupiter and Saturn in the gaseous protoplanetary disk. Icarus 191:158-171

Mordasini C, Alibert Y, Georgy C et al. (2012) Characterization of exoplanets from their formation. II. The planetary mass-radius relationship. A\&A547:A112

Mortier A, Santos NC, Sousa S et al. (2013) On the functional form of the metallicity-giant planet correlation. A\&A551:A112

Morton TD Johnson JA (2011) On the Low False Positive Probabilities of Kepler Planet Candidates. ApJ738:170

Morton TD, Bryson ST, Coughlin JL et al. (2016) False Positive Probabilities for all Kepler Objects of Interest: 1284 Newly Validated Planets and 428 Likely False Positives. ApJ822:86 
Moutou C, Pont F, Barge P et al. (2005) Comparative blind test of five planetary transit detection algorithms on realistic synthetic light curves. A\&A437:355-368

Moutou C, Deleuil M, Guillot T et al. (2013) CoRoT: Harvest of the exoplanet program. Icarus226:1625-1634

Naef D, Mayor M, Beuzit JL et al. (2005) The ELODIE planet search: synthetic view of the survey and its global detection threshold. In: Favata F, Hussain GAJ Battrick B (eds) 13th Cambridge Workshop on Cool Stars, Stellar Systems and the Sun, ESA Special Publication, vol 560, p 833

Nayakshin S (2017) Dawes Review 7: The Tidal Downsizing Hypothesis of Planet Formation. PASA34:e02

Nayakshin S Fletcher M (2015) Tidal Downsizing model - III. Planets from sub-Earths to brown dwarfs: structure and metallicity preferences. MNRAS452:1654-1676

Neveu-VanMalle M, Queloz D, Anderson DR et al. (2016) Hot Jupiters with relatives: discovery of additional planets in orbit around WASP-41 and WASP-47. A\&A586:A93

Pepe F, Molaro P, Cristiani S et al. (2014) ESPRESSO: The next European exoplanet hunter. Astronomische Nachrichten 335:8

Pollacco DL, Skillen I, Collier Cameron A et al. (2006) The WASP Project and the SuperWASP Cameras. PASP118:1407-1418

Pollack JB, Hubickyj O, Bodenheimer P et al. (1996) Formation of the Giant Planets by Concurrent Accretion of Solids and Gas. Icarus 124:62-85

Quinn SN, White RJ, Latham DW et al. (2012) Two "b"s in the Beehive: The Discovery of the First Hot Jupiters in an Open Cluster. ApJ756:L33

Rowe JF, Bryson ST, Marcy GW et al. (2014) Validation of Kepler's Multiple Planet Candidates. III. Light Curve Analysis and Announcement of Hundreds of New Multi-planet Systems. ApJ784:45

Sahu KC, Casertano S, Bond HE et al. (2006) Transiting extrasolar planetary candidates in the Galactic bulge. Nature443:534-540

Santerne A (2014) Professional / Amateur collaborations in exoplanet science. European Planetary Science Congress 2014, EPSC Abstracts, Vol 9, id EPSC2014-188 9:EPSC2014-188

Santerne A, Díaz RF, Moutou C et al. (2012) SOPHIE velocimetry of Kepler transit candidates. VII. A false-positive rate of 35\% for Kepler close-in giant candidates. A\&A545:A76

Santerne A, Moutou C, Tsantaki M et al. (2016) SOPHIE velocimetry of Kepler transit candidates. XVII. The physical properties of giant exoplanets within 400 days of period. A\&A587:A64

Santos NC, Israelian G Mayor M (2001) The metal-rich nature of stars with planets. A\&A373:1019-1031

Santos NC, Israelian G, Mayor M, Rebolo R Udry S (2003) Statistical properties of exoplanets. II. Metallicity, orbital parameters, and space velocities. A\&A398:363-376

Santos NC, Sousa SG, Mortier A et al. (2013) SWEET-Cat: A catalogue of parameters for Stars With ExoplanETs. I. New atmospheric parameters and masses for 48 stars with planets. A\&A556:A150

Schlaufman KC Winn JN (2016) The Occurrence of Additional Giant Planets Inside the Water-Ice Line in Systems with Hot Jupiters: Evidence Against High-Eccentricity Migration. ApJ825:62

Schneider J, Dedieu C, Le Sidaner P, Savalle R Zolotukhin I (2011) Defining and cataloging exoplanets: the exoplanet.eu database. A\&A532:A79

Sousa SG, Santos NC, Israelian G, Mayor M Udry S (2011) Spectroscopic stellar parameters for 582 FGK stars in the HARPS volume-limited sample. Revising the metallicity-planet correlation. A\&A533:A141

Udry S Santos NC (2007) Statistical Properties of Exoplanets. ARA\&A45:397-439

Udry S, Mayor M Santos NC (2003) Statistical properties of exoplanets. I. The period distribution: Constraints for the migration scenario. A\&A407:369-376

Valsecchi F, Rasio FA Steffen JH (2014) From Hot Jupiters to Super-Earths via Roche Lobe Overflow. ApJ793:L3

Vidal-Madjar A, Lecavelier des Etangs A, Désert JM et al. (2003) An extended upper atmosphere around the extrasolar planet HD209458b. Nature422:143-146 
Weiss LM, Marcy GW, Rowe JF et al. (2013) The Mass of KOI-94d and a Relation for Planet Radius, Mass, and Incident Flux. ApJ768:14

Winn JN, Sanchis-Ojeda R, Rogers L et al. (2017) Absence of a metallicity effect for ultra-shortperiod planets. ArXiv e-prints

Wright JT, Marcy GW, Howard AW et al. (2012) The Frequency of Hot Jupiters Orbiting nearby Solar-type Stars. ApJ753:160 